\title{
RECENT ADVANCES IN SYMPLECTIC FLEXIBILITY
}

\author{
YAKOV ELIASHBERG
}

\begin{abstract}
Flexible and rigid methods coexisted in symplectic topology from its inception. While the rigid methods dominated the development of the subject during the last three decades, the balance has somewhat shifted to the flexible side in the last three years. In the talk we survey the recent advances in symplectic flexibility in the work of S. Borman, K. Cieliebak, T. Ekholm, E. Murphy, I. Smith, and the author.
\end{abstract}

This survey article is an expanded version of author's article 27] and his talk at the Current Events Bulletin at the AMS meeting in Baltimore in January 2014. It also uses materials from the papers $[6,9,20,32$ and the book [8].

\section{THE $h$-PRINCIPLE}

Many problems in mathematics and its applications deal with partial differential equations, partial differential inequalities or, more generally, with partial differential relations (see [48] and also [31]), i.e., any conditions imposed on partial derivatives of an unknown function. A solution of such a partial differential relation $\mathcal{R}$ is any function which satisfies this relation.

With any differential relation one can associate the underlying algebraic relation by substituting all the derivatives entering the relation with new independent functions. The existence of a solution of the corresponding algebraic relation, called a formal solution of the original differential relation $\mathcal{R}$, is a necessary condition for the solvability of $\mathcal{R}$. Though it seems that this necessary condition should be very far from being sufficient, it was a surprising discovery in the 1950s of geometrically interesting problems where existence of a formal solution is the only obstruction for the genuine solvability. Two of the first such non-trivial examples were the $C^{1}$ isometric embedding theorem of J. Nash and N. Kuiper [57,68 and the immersion theory of S. Smale and M. Hirsch [55,77. After Gromov's remarkable series of papers, beginning with his paper [47] and culminating in his book [48, the area crystallized as an independent subject, called the $h$-principle.

Rigid and flexible results coexist in many areas of geometry, but nowhere else do they come so close to each other, as in symplectic topology, which serves as a rich source of examples on both sides of the flexible-rigid spectrum. Flexible and rigid problems and the development of each side toward the other shaped and continue to shape the subject of symplectic topology from its inception.

Received by the editors May 9, 2014.

2010 Mathematics Subject Classification. Primary 53D10, 53D05, 53D12.

The author was supported in part by NSF Grant DMS-1205349. 


\section{SyMPLECTIC PRELIMINARIES}

To set the stage, we recall some basic notions of symplectic and contact geometry. Symplectic geometry was born as a geometric language of classical mechanics and, similarly, contact geometry emerged as a natural set-up for geometric optics and mechanics with non-holonomic constraints.

The cotangent bundle $T^{*} M$ of any smooth $n$-dimensional manifold $M$ carries a canonical Liouville 1-form $\lambda$, usually denoted $p d q$, which in any local coordinates $\left(q_{1}, \ldots, q_{n}\right)$ on $M$ and dual coordinates $\left(p_{1}, \ldots, p_{n}\right)$ on cotangent fibers can be written as $\lambda=\sum_{1}^{n} p_{i} d q_{i}$. The differential $\omega:=d \lambda=\sum_{1}^{n} d p_{i} \wedge d q_{i}$ is called the canonical symplectic structure on the cotangent bundle $T^{*} M$. In the Hamiltonian formalism of classical mechanics the cotangent bundle $T^{*} M$ is viewed as the phase space of a mechanical system with the configuration space $M$, where the $p$-coordinates correspond to momenta. The full energy of the system, expressed through coordinates and momenta, i.e., viewed as a function $H: T^{*} M \rightarrow \mathbb{R}$ on the cotangent bundle (or a time-dependent family of functions $H_{t}: T^{*} M \rightarrow \mathbb{R}$ if the system is not conservative) is called the Hamiltonian of the system. The dynamics is then defined by the Hamiltonian equations $\dot{z}=X_{H_{t}}(z), z \in T^{*} M$, where the Hamiltonian vector field $X_{H_{t}}$ is determined by the equation $i\left(X_{H_{t}}\right) \omega=-d H_{t}$, which in the canonical $(p, q)$-coordinates has the form

$$
X_{H_{t}}=\sum_{1}^{n}-\frac{\partial H_{t}}{\partial q_{i}} \frac{\partial}{\partial p_{i}}+\frac{\partial H_{t}}{\partial p_{i}} \frac{\partial}{\partial q_{i}} .
$$

The flow of the vector field $X_{H_{t}}$ preserves $\omega$, i.e., $X_{H_{t}}^{*} \omega=\omega$. The isotopy generated by the vector field $X_{H_{t}}$ is called Hamiltonian.

More generally, the Hamiltonian dynamics can be defined on any $2 n$-dimensional manifold endowed with a symplectic, i.e., a closed and non-degenerate differential 2 -form $\omega$. According to a theorem of Darboux, any such form admits local canonical coordinates $p_{1}, \ldots, p_{n}, q_{1}, \ldots, q_{n}$ in which it can be written as $\omega=\sum_{1}^{n} d p_{i} \wedge d q_{i}$. Diffeomorphisms preserving $\omega$ are called symplectomorphisms or, in the mechanical context, canonical transformations. Symplectomorphisms which can be included in a time-dependent Hamiltonian flow are called Hamiltonian. When $n=1$, a symplectic form is just an area form, and symplectomorphisms are area-preserving transformations. In higher dimensions symplectomorphisms are also volume preserving, but the subgroup of symplectomorphisms represents only a small part of the group of volume-preserving diffeomorphisms.

Contact geometry is an odd-dimensional counterpart of symplectic geometry. The projectivized cotangent bundle $P T^{*} M$ serves as the phase space in geometric optics, because even in the anisotropic media the speed of light is determined by the direction of the ray. It can be interpreted as the space of contact elements of the manifold $M$, i.e., the space of all tangent hyperplanes to $M$. The form $p d q$ does not descend to $P T^{*} M$ but its kernel does, and hence the space of contact elements carries a canonical field of tangent hyperplanes. This field turns out to be completely non-integrable. It is called a contact structure. More generally, a contact structure on a $(2 n+1)$-dimensional manifold is a completely non-integrable field of tangent hyperplanes $\xi$, where the complete non-integrability can be expressed by the Frobenius condition $\alpha \wedge(d \alpha)^{\wedge n} \neq 0$ for a 1-form $\alpha$ (locally) defining $\xi$ by the Pfaffian equation $\alpha=0$. By Darboux's theorem the contact form $\alpha$ can be locally always written in the form $\alpha=d z+\sum_{1}^{n} p_{i} d q_{i}$ in appropriate local coordinates. 
Though at first glance symplectic and contact geometries are quite different, they are in fact tightly interlinked and it is useful to study them in parallel.

An important property of symplectic and contact structures is the following stability theorem, which is due to Moser 65] in the symplectic case and to Gray [44] in the contact one: Given a 1-parametric family of symplectic structures $\omega_{t}$, or contact structures $\xi_{t}$ on a manifold $X$, which coincide outside of a compact set and such that in the symplectic case $\omega_{t}-\omega_{0}$ belong to the same cohomology class with compact support, there exists an isotopy $h_{t}: X \rightarrow X$ with compact support which starts at the identity $h_{0}=\operatorname{Id}$ and such that $h_{t}^{*} \omega_{t}=\omega_{0}$ or $h_{t}^{*} \xi_{t}=\xi_{t}$.

Maximal integral (i.e., tangent to $\xi$ ) submanifolds of a $(2 n+1)$-dimensional contact manifold $(V, \xi)$ have dimension $n$ and are called Legendrian. Their symplectic counterparts are $n$-dimensional submanifolds $L$ of a $2 n$-dimensional symplectic manifold $(W, \omega)$ which are isotropic for $\omega$, i.e., $\left.\omega\right|_{L}=0$. They are called Lagrangian submanifolds. Here are two important examples of Lagrangian submanifolds: A diffeomorphism $f: W \rightarrow W$ of a symplectic manifold $(W, \omega)$ is symplectic if and only if its graph $\Gamma_{f}=\{(x, f(x)) ; x \in W\} \subset(W \times W, \omega \times(-\omega))$ is Lagrangian. A 1-form $\theta$ on a manifold $M$, viewed as a section of the cotangent bundle $T^{*} M$, is Lagrangian if and only if it is closed. In particular, if $H_{1}(M)=0$, then Lagrangian sections are graphs of differentials of functions, and hence the intersection points of a Lagrangian with the 0 -section are critical points of the corresponding generating function. A general Lagrangian submanifold corresponds to a multivalued function, called the front of the Lagrangian manifold. Given a submanifold $N \subset M$ (of any codimension), the set of all tangent to $N$ hyperplanes in $T M$ is a Legendrian submanifold of the space of contact elements $P T^{*} M$.

\section{Gromov's Alternative AND Discovery of SYMPLECTIC RIGIDITY}

It was an original idea of Henri Poincaré that Hamiltonian systems should satisfy special qualitative properties. In particular, his study of periodic orbits in the so-called restricted three-body problem led him to the following statement, now known as the "last geometric theorem of H. Poincaré": any area-preserving transformation of an annulus $S^{1} \times[0,1]$ which rotates the boundary circles in opposite directions should have at least two fixed points. Poincaré provided many convincing arguments why the statement should be true [74], but the actual proof was found by G. D. Birkhoff [5] in 1913, a few months after Poincaré's death. Birkhoff's proof was purely 2-dimensional, and further development of Poincaré's dream of what is now called symplectic topology had to wait until the 1960s when V. I. Arnold [3] formulated a number of conjectures formalizing this vision of Poincaré. In particular, one of Arnold's conjectures stated that the number of fixed points of a Hamiltonian diffeomorphism is bounded below by the minimal number of critical points of a function on the symplectic manifold.

At about the same time Gromov was proving his $h$-principle type results. He realized that symplectic problems exhibited some remarkable flexibility. This called into question whether Arnold's conjectures could be true in dimension $>2$.

Among the remarkable results pointing toward symplectic flexibility which were proven by Gromov at the end of the 1960s and the beginning of the 1970s were the following:

- $h$-principle for symplectic and contact structures on open manifolds: In any homotopy class of non-degenerate (not necessarily closed) 2-forms on 
an open manifold there is a symplectic form in any prescribed cohomology class. Moreover, any two such forms are homotopic as symplectic forms. Similarly, any almost contact structure, i.e., a pair $(\lambda, \eta)$ of 1 - and 2 -forms on a $(2 k+1)$-dimensional open manifold which satisfies $\lambda \wedge \eta^{k} \neq 0$, is homotopic through almost contact structures to a pair $(\alpha, d \alpha)$.

- $h$-principle for Lagrangian immersions: This asserts that the Lagrangian regular homotopy classes of Lagrangian immersions $L \rightarrow X$ are in one-toone correspondence with homotopy classes of injective Lagrangian homomorphisms $T L \rightarrow T X$.

- $h$-principle for $\epsilon$-Lagrangian embeddings (i.e., embeddings whose tangent planes deviate from Lagrangian directions by an angle $<\epsilon$ ).

- $h$-principle for the iso-symplectic and iso-contact embeddings: For instance, in the symplectic case, if $(M, \omega)$ and $(N, \eta)$ are two symplectic manifolds such that $\operatorname{dim} N \geq \operatorname{dim} M+4$, then any smooth embedding $f: M \rightarrow N$ which pulls back the cohomology class of the form $\eta$ to the cohomology class of $\omega$ and whose differential df is homotopic to a symplectic bundle isomorphism can be $C^{0}$-approximated by an iso-symplectic embedding $\tilde{f}: M \rightarrow N$, i.e. $\tilde{f}^{*} \eta=\omega$. For iso-symplectic and iso-contact immersions the $h$-principle holds even in codimension 2 .

Gromov proved the following alternative: either the group of symplectomorphisms (resp. contactomorphisms) is $C^{0}$-closed in the group of all diffeomorphisms or its $C^{0}$-closure coincides with the group of volume-preserving (resp. all) diffeomorphisms.

One of the corollaries of Gromov's convex integration method was that there are no additional lower bounds for the number of fixed points of a volume-preserving diffeomorphism of a manifold of dimension $\geq 3$ (compared to arbitrary diffeomorphisms). Clearly, the bound on the number of fixed points is a $C^{0}$-property, and hence, if the second part of the alternative were true, this would imply that Hamiltonian diffeomorphisms of symplectic manifolds of dimension $>2$ have no special fixed point properties, and hence Poincaré's theorem and Arnold's conjectures reflected a pure 2-dimensional phenomenon. In fact, it was clear from this alternative, that all basic problems of symplectic topology are tightly interconnected.

Here are some of such problems, besides Gromov's alternative:

Problems 3.1. (1) Extension of symplectic and contact structures to the ball from a neighborhood of the boundary sphere.

(2) 1-parametric version of the previous question: Is it true that two structures on the ball which coincide near the boundary and which are formally homotopic relative to the boundary are homotopic as genuine structures and hence isotopic?

(3) Fixed point problems for symplectomorphisms. More generally, Lagrangian intersection problem: Do Lagrangian manifolds under certain conditions have more intersection points than is required by topology?

(4) Are there any non-formal obstructions to Legendrian isotopy?

For instance, as was already pointed out above, the resolution of Gromov's alternative in favor of flexibility would imply that symplectic fixed point Arnold conjectures are wrong in dimension $>2$. Similarly, the "flexible" resolution of both problems (1) and (2) would imply the "flexible" resolution of Gromov's alternative, 
as we explain below. Thus, a resolution of Gromov's alternative became a question about the existence of symplectic topology as a subject.

At the beginning of the 1980s the alternative was resolved in favor of rigidity in the series of works [21, 22, 4, [12, and it culminated in Gromov's paper 46] in which he introduced his method of (pseudo-)holomorphic curves in symplectic manifolds, which brought a genuine revolution into this subject.

As we already stated above, the rigid resolution of the symplectic and contact alternatives implied that Problems 3.1 (1) and 3.1 (2) cannot simultaneously have flexible solutions. Indeed, consider, for instance, the contact case. Let $(M, \xi)$ be a $(2 n+1)$-dimensional contact manifold, and let $f: M \rightarrow M$ be isotopic to the identity diffeomorphism which is somewhere not contact. Let us first note that according to Gromov's $h$-principle for open contact manifolds, the analogs of Problems 3.1(1) and 3.1(2) do have positive answers for neighborhoods of discs of positive codimension. Assuming that both questions 3.1 (1) and 3.1 (2) have positive answers as well, we consider a small triangulation of $M$ and inductively modify $f$ by a $C^{0}$-small isotopy to make it contact on neighborhoods of $k$-skeleta of the triangulation, $k=0, \ldots, 2 n+1$. Suppose that we already constructed a diffeomorphism $f_{k-1}: M \rightarrow M$ preserving $\xi$ on a neighborhood of the $(k-1)$ skeleton $C_{k-1}$. Take a neighborhood $G_{\sigma} \supset \sigma$ of a $k$-simplex $\sigma$. Consider a contact structure on $\mathcal{O} p\left(\partial G_{\sigma} \cup \sigma\right) \subset G_{\sigma}$ which coincides with $\xi$ on $\mathcal{O} p \partial G_{\sigma}$ and with $\left(f_{k-1}\right)_{*} \xi$ on $\mathcal{O} p \sigma$. Using a positive resolution of Problem 3.1(1), we can then extend it as a contact structure on $G_{\sigma}$ which belongs to the same $\xi$ relative to $\partial G_{\sigma}$ homotopy class of almost contact structures. Hence, assuming a positive resolution of Problem 3.1 (2) together with a Gray-Moser argument then implies existence of a compactly supported in $G_{\sigma}$ diffeomorphism $g_{\sigma}: M \rightarrow M$ such that $g_{\sigma}^{*} \xi=\widetilde{\xi}$. Note that one can arrange that supports of $g_{\sigma}$ and $g_{\widehat{\sigma}}$ are disjoint if $\sigma \cap \widehat{\sigma}=\varnothing$. Then the composition $g$ of the diffeomorphisms $g_{\sigma}$ for all $k$-simplices $\sigma$ is $C^{0}$-small provided that the triangulation and the neighborhoods $G_{\sigma}$ have small diameters. But then the diffeomorphism $f_{k}:=g \circ f_{k-1}$ is $C^{0}$-close to $f$ and preserves the contact structure $\xi$ on a neighborhood of the $k$-skeleton $C_{k}$. Continuing by induction, we construct a contactomorphism $f_{2 n+1}$ which is $C^{0}$-close to $f$. But this contradicts to the $C^{0}$-closedness of the group of contact diffeomorphisms.

In fact, an argument similar to Gromov's famous non-squeezing theorem also implies that there are additional obstructions to the extendability of symplectic structures; see Section 6.2. This still left open the possibility of a flexible resolution of Problem 3.1 (2) in the symplectic case. The problem remains open today 1 In the contact case by now both problems are answered; see the discussion in Section 5.7 .

After Gromov's paper, the rigid side of symplectic topology began unravelling with an exponentially increasing speed. We just mention here the discovery of Floer homology, Hofer's metric, Gromov-Witten invariants, Symplectic Field Theory, the link with the mathematical theory of Mirror Symmetry, as well applications to lower-dimensional topology such as Taubes's "Gromov-Witten = Seiberg-Witten" theorem, the Heegaard Floer homology of Ozsváth and Szabó, and the embedded contact homology of Hutchings and Taubes.

\footnotetext{
${ }^{1}$ Gromov proved that any standard at infinity symplectic structure on $\mathbb{R}^{4}$ is symplectomorphic to the standard one. Hence, this reduced the isotopy classification of such structures to the computation of $\pi_{0}$ of the group of compactly supported diffeomorphisms of $\mathbb{R}^{4}$.
} 
Applications of holomorphic curves in Hamiltonian dynamics brought us closer to the realization of Poincaré's dream of establishing qualitative properties of mechanical systems (e.g., existence and the number of periodic trajectories) without actually solving the equations of motion. In particular, the Weinstein conjecture, which asserted the existence of periodic trajectories of Reeb vector fields, was proven in many cases (see [56, 86]) and in full generality in dimension 3 (see [80]).

\section{Flexible milestones after the Resolution of Gromov's Alternative}

Though in a shadow of successes on the rigid side, the flexible side of symplectic topology also had over the years a number of success stories. Here are examples of some interesting developments with a distinctly flexible flavor.

Overtwisted contact structures in dimension 3. It was understood in 1989 (see [23) that in the world of 3-dimensional contact manifolds there is an important dichotomy. If a contact manifold contains the so-called overtwisted disc, i.e., an embedded disc which along its boundary is tangent to the contact structure, then the contact structure becomes very flexible and abides by a certain $h$-principle: two overtwisted contact structures which are homotopic as plane fields are homotopic as contact structures, and hence in view of Gray's theorem are isotopic. Nonovertwisted contact manifolds are called tight, and that is where the rigid methods of symplectic topology are applicable.

The classification of overtwisted contact structures yields similar flexibility results for Legendrian knots in overtwisted contact 3-manifolds. Namely, Legendrian knots in the complement of an overtwisted disc (called loose in [28]) also satisfy an $h$-principle. The high-dimensional analog of loose knots is discussed in Section 5.1 below.

It was recently discovered that an analog of classification of overtwisted contact structures holds in all dimensions; see Section 5.7 below.

Donaldson's almost holomorphic sections. We already mentioned above Gromov's $h$-principle for iso-symplectic embeddings in codimension $>2$. By applying holomorphic curve technique, it is not difficult to construct counter-examples to a similar $h$-principle in codimension 2. However, Simon Donaldson used his theory of almost holomorphic sections of complex line bundles over almost Kähler manifolds to prove the following, among other remarkable results:

Theorem 4.1 ([13]). For any closed $2 n$-dimensional symplectic manifold $(M, \omega)$ with an integral cohomology class $[\omega] \in H^{2}(M)$ and a sufficiently large integer $k$, there exists a codimension 2 symplectic submanifold $\Sigma \subset M$ which represents the homology class that is Poincaré dual to kw. Moreover, the complement $M \backslash \Sigma$ has a homotopy type of an n-dimensional cell complex (which is the case for complements of hyperplane sections in complex projective manifolds).

Furthermore, he proved the following symplectic Lefschetz pencil theorem:

Theorem $4.2([15])$. If $(V, \omega)$ is a symplectic manifold with an integral cohomology class $[\omega] \in H^{2}(M)$, then for a sufficiently large integer $k$ there exists a topological Lefshetz pencil in which the fibers are symplectic manifolds representing a homology class dual to $k[\omega]$. 
By definition the topological Lefschetz pencil is equivalent to the complex algebraic one near all the singularities.

E. Giroux [41] adapted Donaldson's theory to the contact case. His theory of open book decompositions of contact manifolds plays an important role in contact topology.

Symplectic embeddings of polydiscs. Let us denote by $P\left(r_{1}, \ldots, r_{n}\right)$ the poly$\operatorname{disc}\left\{\left|z_{1}\right| \leq r_{1}, \ldots,\left|z_{n}\right| \leq r_{n}\right\} \subset \mathbb{C}^{n}$, where we assume $r_{1} \leq r_{2} \leq \cdots \leq r_{n}$. If $P\left(r_{1}, \ldots, r_{n}\right)$ symplectically embeds into $P\left(R_{1}, \ldots, R_{n}\right)$, then the famous Gromov non-squeezing theorem implies that $r_{1} \leq R_{1}$. We also have the volume constraint $r_{1} \cdots r_{n} \leq R_{1} \cdots R_{n}$.

It was a common belief that when $n>2$ there should exist further constraints on the radii besides the Gromov width and volume constraints. However, Larry Guth proved the following remarkable result on the flexible side, which showed that room for additional constraints is very limited.

Theorem 4.3 (49]). There exists a constant $C(n)$ depending on the dimension $n$ such that if $C(n) r_{1} \leq R_{1}$ and $C(n) r_{1} \cdots r_{n} \leq R_{1} \cdots R_{n}$, then a polydisc $P\left(r_{1}, \ldots, r_{n}\right)$ symplectically embeds into $P\left(R_{1}, \ldots, R_{n}\right)$.

4.1. Existence of Stein complex structure. Stein manifolds are complex manifolds which admit proper holomorphic embeddings into $\mathbb{C}^{N}$. According to a theorem of H. Grauert, a Stein manifold can also be characterized as a manifold which admits an exhausting strictly plurisubharmonic function. Here the word $e x-$ hausting means proper and bounded below, while a real-valued function $\phi: V \rightarrow \mathbb{R}$ on a complex manifold $V$ is called strictly plurisubharmonic or $i$-convex if the Hermitian form $-d d^{\mathbb{C}} \phi=2 i \partial d \phi$, which in local holomorphic coordinates is given by a matrix $\left(\frac{\partial^{2} \phi}{\partial z_{i} \partial \bar{z}_{j}}\right)$, is positive definite. For an arbitrary complex manifold with a complex structure $J$, we will use the term $J$-convex, instead of strictly plurisubharmonic, to stress the dependence on the complex structure $J$. Here we denote by $d^{\mathbb{C}} \phi(X):=d \phi(i X)$ the differential twisted by the operator of multiplication by $\sqrt{-1}$. It can easily be seen that critical points of a Morse strictly plurisubharmonic function on a complex $n$-dimensional manifold have index $\leq n$, and hence the Morse theory implies that a Stein manifold of complex dimension $n$ has a homotopy type of a cell complex of real dimension $n$.

Theorem 4.4 ([24]). Let $(V, J)$ be any manifold of dimension $2 n>4$, and let $\phi: V \rightarrow \mathbb{R}$ be an exhausting Morse function without critical points of index $>n$. Then there exists an integrable complex structure $\widetilde{J}$ on $V$ homotopic to $J$ for which the function $\phi$ is target equivalent to a $\widetilde{J}$-convex function. In particular, $(V, \widetilde{J})$ is Stein.

Interestingly, complex dimension 2 belongs to the domain of symplectic rigidity. An analog of Theorem 4.4 is wrong in this dimension. For instance, $S^{2} \times \mathbb{R}^{2}$ does not admit any Stein complex structure; see 59.

What is transpired from the proof of Theorem 4.4 is that it is useful to define a symplectic analog of a Stein manifold. The corresponding notion of Weinstein manifold, crucial for an understanding of Morse theoretic properties of Stein structures, was introduced in [30]. It formalized the Stein handlebody construction from [24] and symplectic handlebody construction from A. Weinstein's paper [88]. We discuss this notion and related results in Section 5.5 below. 


\section{RENAissance OF THE $h$-PRINCIPLE IN SYMPLECTIC TOPOLOGY}

The last three years witnessed a number of quite unexpected advances on the flexible side of symplectic topology.

5.1. Loose Legendrian knots. It turns out that in contact manifolds of dimension $>3$ there is a remarkable class of Legendrian embeddings - or as we also refer to them Legendrian knots, discovered by Emmy Murphy in 67 — which satisfies a certain form of an $h$-principle. These knots are called loose, in analogy with loose knots in overtwisted contact manifolds; see [28. A remarkable fact about Murphy's loose knots is that, in contrast with the 3 -dimensional case, they exist in all contact manifolds of dimension $>3$.

Stabilization. The stabilization construction for Legendrian submanifolds, first introdiced in 24 and also described in [8,67, can be informally defined as follows. In an appropriate Darboux coordinate system near a point on a Legendrian submanifold $\Lambda$, its front projection has a cuspidal edge; see Figure 5.1. Then given a domain $U$ near a cusp edge on the lower branch of the front, we push it up so over $U$ the lower branch crosses over the upper one. The Legendrian submanifold corresponding to the modified front is called the $U$-stabilization of $\Lambda$.

More precisely, in the standard contact

$$
\mathbb{R}_{\mathrm{st}}^{2 n-1}=\left(\mathbb{R}^{2 n-1}, \xi_{\mathrm{st}}=\operatorname{ker}\left(d z+\sum_{1}^{n-1} p_{i} d q_{i}\right)\right),
$$

where $\left(p_{1}, q_{1}, \ldots, p_{n-1}, q_{n-1}, z\right)$ are coordinates in $\mathbb{R}^{2 n-1}$, consider a diffeomorphic to $\mathbb{R}^{n-1}$ Legendrian submanifold

$$
\Lambda_{\mathrm{cu}}=\left\{\left(p_{1}, q_{1}, \ldots, p_{n-1}, q_{n-1}, z\right): q_{1}=\frac{1}{2} p_{1}^{2}, q_{2}=\cdots=q_{n-1}=0, z=-\frac{1}{3} p_{1}^{3}\right\} .
$$

One can check that given any Legendrian $(n-1)$-submanifold $\Lambda \subset Y$ in a contact $(2 n-1)$-manifold $Y$, any point $u \in \Lambda$ has an arbitrarily small neighborhood $\Omega \subset Y$ that admits a contactomorphism

$$
\Phi:(\Omega, \Lambda \cap \Omega) \rightarrow\left(\mathbb{R}_{\mathrm{st}}^{2 n-1}, \Lambda_{\mathrm{cu}}\right), \quad \Phi(u)=0 .
$$

The stabilization construction is a local modification of a Legendrian knot in a neighborhood of a point. It replaces the preimage of $\Lambda_{\text {cu }}$ by a preimage of another Legendrian $\Lambda_{\mathrm{cu}}^{U}$, which coincides with $\Lambda_{\mathrm{cu}}$ at infinity. We describe this modification below.

The two branches of the front $\Gamma_{\mathrm{cu}}$ of the Legendrian $\Lambda_{\mathrm{cu}}$, i.e., the projection to the $\left(x_{1}, \ldots, x_{n-1}, z\right)$-coordinate subspace, are graphs of the functions $\pm h$, where

$$
h(q)=h\left(q_{1}, \ldots, q_{n-1}\right)=\frac{2 \sqrt{2}}{3} q_{1}^{\frac{3}{2}},
$$

defined on the half-space $\mathbb{R}_{+}^{n-1}:=\left\{q=\left(q_{1}, \ldots, q_{n-1}\right): q_{1} \geq 0\right\}$. Let $U$ be a domain with smooth boundary contained in the interior of $\mathbb{R}_{+}^{n-1}, U \subset \operatorname{Int}\left(\mathbb{R}_{+}^{n-1}\right)$. Pick a non-negative function $\phi: \mathbb{R}_{+}^{n+1} \rightarrow \mathbb{R}$ with the following properties:

- $\phi$ has compact support in Int $\left(\mathbb{R}_{+}^{n-1}\right)$;

- the function $\widetilde{\phi}(q):=\phi(q)-2 h(q)$ is Morse, $U=\widetilde{\phi}^{-1}([0, \infty))$, and 0 is a regular value of $\widetilde{\phi}$. 


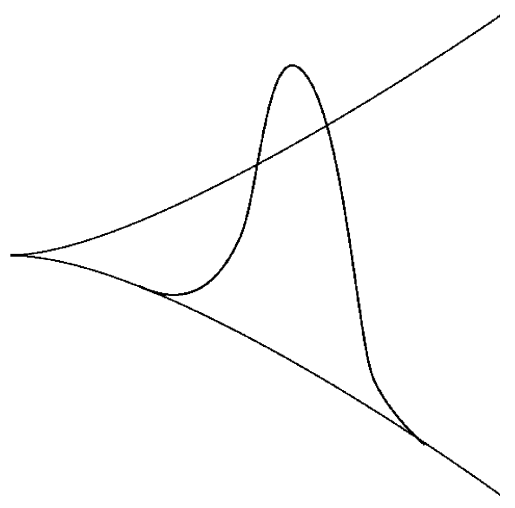

FIGURE 5.1. Stabilization

Consider the front $\Gamma_{\mathrm{cu}}^{U}$ in $\mathbb{R}^{n-1} \times \mathbb{R}$ obtained from $\Gamma_{\mathrm{cu}}$ by replacing the lower branch of $\Gamma_{\text {cu }}$, i.e., the graph $z=-h(q)$, by the graph $z=\phi(q)-h(q)$. Since $\phi$ has compact support, the front $\Gamma_{\mathrm{cu}}^{U}$ coincides with $\Gamma_{\mathrm{cu}}$ outside a compact set. Consequently, the Legendrian embedding $\Lambda_{\text {cu }}^{U}: \mathbb{R}^{n-1} \rightarrow \mathbb{R}^{2 n-1}$ defined by the front $\Gamma_{\text {cu }}^{U}$ coincides with $\Lambda_{\text {cu }}$ outside a compact set.

It turns out that if (and only if) the Euler characteristic of the domain $U$ is equal to 0 , then the Legendrian submanifolds $\Lambda_{\mathrm{cu}}$ and $\Lambda_{\mathrm{cu}}^{U}$ are formally Legendrian isotopic via a compactly supported Legendrian isotopy (however, they are never Legendrian isotopic if $U \neq \varnothing)$. We recall that a formal Legendrian isotopy connecting Legendrian embeddings $f_{0}, f_{1}: \Lambda \rightarrow(Y, \xi)$ is a pair $f_{t}, \Phi_{t}, t \in[0,1]$, where $f_{t}$ is a smooth isotopy and $\Phi_{t}: T \Lambda \rightarrow \xi$ is a family of Lagrangian homomorphisms connecting $\phi_{0}=d f_{0}$ and $\Phi_{1}=d f_{1}$, and such that the paths of homomorphisms $d f_{t}, \Phi_{t}$ are homotopic with fixed endpoints as paths of injective homomorphisms $T \Lambda \rightarrow T Y$. We also note that when $n=1$, the Euler characteristic $\chi(U)$ is always positive, and hence the stabilization construction never preserves the formal isotopy class of a 1-dimensional Legendrian knot. This is the main point where the theory in high dimensions deviates from the 1-dimensional case.

Now, given a Legendrian $(n-1)$-submanifold $\Lambda$ of a contact $(2 n-1)$-manifold $Y$ and contactomorphism

$$
\Phi:(\Omega, \Lambda \cap \Omega) \rightarrow\left(\mathbb{R}_{\mathrm{st}}^{2 n-1}, \Lambda_{\mathrm{cu}}\right),
$$

$\Omega \subset Y$ is a neighborhood of a point of $u \in \Lambda$, and we replace $\Omega \cap \Lambda$ with $\Phi^{-1}\left(\Lambda_{\mathrm{cu}}^{U}\right)$. The resulting Legendrian embedding $\Lambda^{U}$ which coincides with $\Lambda$ outside of $\Omega$ is called the $U$-stabilization of $\Lambda$ in $\Omega$.

5.2. Murphy's theorem. A Legendrian embedding $\Lambda \rightarrow Y$ of a connected manifold $\Lambda$ is called loose if it is isotopic to the stabilization of another Legendrian knot. We point out that looseness depends on the ambient manifold. A loose Legendrian embedding $\Lambda$ into a contact manifold $Y$ need not be loose in a smaller neighborhood $Y^{\prime}, \Lambda \subset Y^{\prime} \subset Y$.

The above construction shows that a Legendrian submanifold $\Lambda \subset Y$ can be made loose by stabilizing it in an arbitrarily small neighborhood of a point and even without changing its formal Legendrian isotopy class. 
It was known since the early days of the $h$-principle that formally isotopic Legendrian knots become isotopic after sufficiently many stabilizations. In dimension 3 the corresponding proof was carried out in [50]. Moreover, it was shown by J. Etnyre and K. Honda in 34 that no a priori given number of stabilizations of 1-dimensional Legendrian knots is sufficient.

It was an unexpected discovery of E. Murphy that in dimension $>1$ one stabilization is always enough. Namely, she proved the following $h$-principle for loose Legendrian knots in contact manifolds of dimension $2 n-1>3$ :

Theorem 5.1 ([67]). Any two loose Legendrian embeddings which coincide outside a compact set and which can be connected by a formal compactly supported Legendrian isotopy can be connected by a genuine compactly supported Legendrian isotopy.

5.3. Lagrangian caps. Murphy's discovery was followed by a number of other results, which seemed to be out of reach before that. In particular, it turned out that Lagrangian embeddings with loose Legendrian boundaries also satisfy an $h$ principle.

The story begins with the following question: Let $B$ be the round ball in the standard symplectic $\mathbb{R}^{2 n}$. Is there an embedded Lagrangian disc $\Delta \subset \mathbb{R}^{2 n} \backslash \operatorname{Int} B$ with $\partial \Delta \subset \partial B$ such that $\partial \Delta$ is a Legendrian submanifold and $\Delta$ transversely intersects $\partial B$ along its boundary?

If $n=2$, then such a Lagrangian disc does not exist: its existence contradicts the so-called slice Bennequin inequality; see [75]. Until recently no such examples were known in higher dimensions either. Surprisingly, it turns out that when $n>2$, then Lagrangian discs with loose Legendrian boundaries satisfy an $h$-principle, which, in particular, implies that they exist in abundance:

Theorem 5.2 ([32]). Let $L$ be a smooth manifold of dimension $n>2$ with nonempty boundary such that its complexified tangent bundle $T L \otimes \mathbb{C}$ is trivial. Then there exists an exact Lagrangian embedding $f:(L, \partial L) \rightarrow\left(\mathbb{R}^{2 n} \backslash \operatorname{Int} B, \partial B\right)$ with $f(\partial L) \subset \partial B$ such that $f(\partial L) \subset \partial B$ is a Legendrian submanifold and $f$ is transverse to $\partial B$ along its boundary $\partial L$.

Note that the triviality of the bundle $T L \otimes \mathbb{C}$ is a necessary (and, according to Gromov's $h$-principle for Lagrangian immersions [4], sufficient) condition for existence of any Lagrangian immersion $L \rightarrow \mathbb{C}^{n}$.

Given a symplectic manifold $(X, \omega)$, we say that $L \subset M$ is a Lagrangian submanifold with an isolated conical point if it is a Lagrangian submanifold away from a point $p \in L$, and there exists a symplectic embedding $f: B_{\varepsilon} \rightarrow X$ such that $f(0)=p$ and $f^{-1}(L) \subset B_{\varepsilon}$ is a Lagrangian cone. Here $B_{\varepsilon}$ is the ball of radius $\varepsilon$ in the standard symplectic $\mathbb{R}^{2 n}$. Note that this cone is automatically a cone over a Legendrian sphere in the sphere $\partial B_{\varepsilon}$ endowed with the standard contact structure given by the restriction to $\partial B_{\varepsilon}$ of the Liouville form $\lambda_{\text {st }}=\frac{1}{2} \sum_{1}^{n}\left(p_{i} d q_{i}-q_{i} d p_{i}\right)$. For instance, Givental's Lagrangian Whitney umbrella (see 43]) can be viewed as a Lagrangian cone over the simplest stabilization of the trivial Legendrian knot.

As a special case of Theorem 5.2 (when $\partial L$ is a sphere) one has

Corollary 5.3 ([32]). Let $L$ be an n-dimensional, $n>2$, closed manifold such that the complexified tangent bundle $T^{*}(L \backslash p) \otimes \mathbb{C}$ is trivial. Then $L$ admits an exact Lagrangian embedding into $\mathbb{R}^{2 n}$ with exactly one conical point. In particular, 
an $n$-sphere admits a Lagrangian embedding to $\mathbb{R}^{2 n}$ with one conical point for each $n>2$.

5.4. Lagrangian non-intersections. The conical singularity with appropriate loose Legendrian asymptotics in Corollary 5.3 can be resolved into an immersion with one self-intersection point. This leads to surprising constructions of Lagrangian immersions with a minimal number of self-intersection points, which at first glance are going against popular Arnold type Lagrangian intersection conjectures. In particular,

Theorem 5.4 (20]). Let $L$ be an $n$-dimensional closed manifold with trivial bundle $T L \otimes \mathbb{C}$. We denote by $s(L)$ the minimal number of double points of a Lagrangian immersion of $L$ into the standard symplectic $\mathbb{R}^{2 n}$. Then the following hold:

(1) If $n$ is odd or if $L$ is non-orientable, then $s(L) \in\{1,2\}$.

(2) If $n=3$, then $s(L)=1$.

(3) If $n$ is even and $L$ is orientable, then for $\chi(L)<0, s(L, \sigma)=\frac{1}{2}|\chi(L)|$, and for $\chi(L) \geq 0$, either $s(L)=\frac{1}{2} \chi(L)$ or $s(L)=\frac{1}{2} \chi(L)+2$.

The case $n=2$ is due to D. Sauvaget 78. It is interesting to compare Theorem 5.4 with the results of [17,19] which show that if $n$ is even, then the standard $n$-sphere is the only homotopy $n$-sphere that admits a self-transverse Lagrangian immersion into Euclidean space with only one double point. This means, in particular, that when $\operatorname{dim}(L)$ is even and $\chi(L)>0$, then $s(L)$ is generally not determined by the homotopy type of $L$. The following result constrains the homotopy type of a manifold for which this phenomenon may occur.

Theorem $5.5([20])$. Let $L$ be an even dimensional spin manifold with $\chi(L)>0$. If $s(L)=\frac{1}{2} \chi(L)$, then $\pi_{1}(L)=1$ and $H_{2 k+1}(L)=0$ for all $k$. In particular if $\operatorname{dim} L>4$, then $L$ has the homotopy type of a $C W$-complex with $\chi(L)$ evendimensional cells and no odd-dimensional cells.

It is interesting to note that even for the standard odd-dimensional sphere $S^{2 k+1}$ the construction in Theorem 5.4 provides an immersion with a single double point of Maslov index 1, which is different from the standard Whitney Lagrangian immersion $S^{2 k+1} \rightarrow \mathbb{R}^{4 k+2}$, where the intersection point has index $2 k+1$. Using Polterovich's surgery 82 we then get

Corollary 5.6. There exists a Lagrangian embedding $S^{1} \times S^{2 k} \rightarrow \mathbb{R}_{\mathrm{st}}^{4 k+2}$ for which the generator of the first homology of positive action has non-positive Maslov index $2-2 k$. In particular, there exists a Lagrangian embedding $S^{1} \times S^{2} \rightarrow \mathbb{R}_{\mathrm{st}}^{6}$ with zero Maslov class.

The problem of possible values of Maslov class for Lagrangian embeddings of $S^{1} \times S^{n-1} \rightarrow \mathbb{R}^{2 n}$ was first discussed by Polterovich in [81,82, who also constructed for an even $n$ a Lagranigian embedding $S^{1} \times S^{n-1} \rightarrow \mathbb{R}^{2 n}$ with Maslov classes $n-1$, in addition to the standard Lagrangian embedding with Maslov class 2, which one gets by resolving the double point of the standard Whitney Lagrangian immersion $S^{n} \rightarrow \mathbb{R}^{2 n}$. For an odd $n$ the only previously known Lagrangian embedding was the standard embedding with Maslov class 2. We also note that Fukaya, Oh, Ohta, and Ono proved (see [51]) that the only possible Maslov class values for Lagranigian embeddings $S^{1} \times S^{n-1} \rightarrow \mathbb{R}^{2 n}$ for $n$ odd are 2 and $2-2 n$. 


\subsection{Flexible Stein and Weinstein manifolds.}

5.5.1. Stein and Weinstein manifolds. Weinstein manifolds, which we define below, are symplectic counterparts of Stein complex manifolds. Their symplectic tpoplogy is another playground where the theory of loose Legendrian knots yields interesting applications.

Definition. A Weinstein structure on an open manifold $V$ is a triple $(\omega, X, \phi)$, where

- $\omega$ is a symplectic form on $V$;

- $\phi: V \rightarrow \mathbb{R}$ is an exhausting Morse (or generalized Morse, i.e., having either non-degenerate or birth-death critical points) function;

- $X$ is a complete vector field which is Liouville for $\omega$ (i.e., $L_{X} \omega=\omega$ ) and gradient-like for the function $\phi$.

The quadruple $(V, \omega, X, \phi)$ is then called a Weinstein manifold, and the function $\phi$ is called a Lyapunov function for the Liouville field $X$.

We note that though any Weinstein structure $(\omega, X, \phi)$ can be perturbed to make the function $\phi$ Morse, in 1-parameter families of Weinstein structures birth-death points are generically unavoidable.

We will also consider Weinstein cobordism structures. Let $W$ be a compact manifold with boundary $\partial W=\partial_{+} W \amalg \partial_{-} W$. A Morse (or generalized Morse) function $\phi: W \rightarrow \mathbb{R}$ is called defining if $\partial_{ \pm} W$ are regular level sets of $\phi$ with $\left.\phi\right|_{\partial_{-} W}=\min \phi$ and $\left.\phi\right|_{\partial_{+} W}=\max \phi$. The notion of a Weinstein cobordism $(W, \omega, X, \phi)$ differs from that of a Weinstein manifold only in replacing the condition that $\phi$ is exhausting by the requirement that $\phi$ is a defining function, and by replacing the completeness condition of $X$ by the requirement that $X$ is inward transverse to $\partial_{-} W$ and outward transverse to $\partial_{+} W$. A Weinstein cobordism with $\partial_{-} W=\emptyset$ is called a Weinstein domain.

In the complex geometric context, let us recall that a $J$-convex (see Section 4.1) function $\phi: V \rightarrow \mathbb{R}$ on a complex manifold $(V, J)$ serves as a potential of a Kähler metric $H_{J, \phi}(X, Y):=g_{J, \phi}(X, Y)-i \omega_{J, \phi}(X, Y)$, where $\omega_{J, \phi}=-d d^{\mathbb{C}} \phi$ and $g_{J, \phi}(X, Y)=\omega_{J, \phi}(X, J Y)$. The gradient $X_{J, \phi}:=\nabla_{J, \phi} \phi$ of the function $\phi$ with respect to the metric $g_{J, \phi}$ is a Liouville field for $\omega_{J, \phi}$, i.e., $L_{X J, \phi} \omega_{J, \phi}=\omega_{J, \phi}$. If $(V, J)$ is Stein, then for any exhausting $J$-convex function $\phi: V \rightarrow \mathbb{R}$ the vector field $X_{J, \phi}$ can be made complete by composing $\phi$ with any function $h: \mathbb{R} \rightarrow \mathbb{R}$ with positive first and second derivatives; see [8]. Assuming that this was already done, we associate with a Stein complex manifold $(V, J)$ together with an exhausting $J$ convex (generalized) Morse function $\phi: V \rightarrow \mathbb{R}$ a Weinstein structure $\mathfrak{W}(V, J, \phi)=$ $\left(V, \omega_{\phi, J}, X_{J, \phi}, \phi\right)$.

By a Stein cobordism structure on a cobordism $W$, we understand a pair $(J, \phi)$ where $J$ is an integrable complex structure on $W$ and $\phi: W \rightarrow \mathbb{R}$ a defining $J$ convex function. A Stein cobordism with empty $\partial_{-} W$ is called a Stein domain. As in the manifold case, any Stein cobordism structure $(J, \phi)$ on $W$ determines a Weinstein cobordism structure $\mathfrak{W}(J, \phi)=\left(W, \omega_{J, \phi}, X_{J, \phi}, \phi\right)$.

The following result is an upgrade of Theorem 4.4 
Theorem $5.7([8])$.

(1) Let $\mathfrak{W}=(V, \omega, X, \phi)$ be a Weinstein structure. Then there exists

- an integrable complex structure $J$ on $V$ for which $\phi$ is $J$-convex and

- a homotopy of Weinstein structures $\mathfrak{W}_{t}=\left(\omega_{t}, X_{t}, \phi\right)$ connecting $\mathfrak{W}_{0}=$ $\mathfrak{W}$ and $\mathfrak{W}_{1}=\mathfrak{W}(V, J, \phi)$.

(2) Let $V$ be a manifold of dimension $2 n \neq 4$, let $\phi: V \rightarrow \mathbb{R}$ be an exhausting Morse function without critical points of index $>n$, and let $\eta$ be a nondegenerate 2 -form. Then there exists a Weinstein structure $(\omega, X, \phi)$ on $V$ such that $\omega$ and $\eta$ are homotopic as non-degenerate forms.

A similar result holds in the cobordism case.

5.5.2. Flexibility. Each Weinstein manifold or cobordism can be cut along regular level sets of its Lyapunov function $\phi$ into Weinstein cobordisms that are elementary in the sense that there are no trajectories of the Liouville vector field connecting different critical points of $\phi$. An elementary $2 n$-dimensional Weinstein cobordism $(W, \omega, X, \phi), n>2$, is called flexible if the attaching spheres of all index $n$ handles form in $\partial_{-} W$ a loose Legendrian link, i.e., each of its components is loose in the complements of the others. A Weinstein cobordism or manifold structure $(\omega, X, \phi)$ is called flexible if it can be decomposed into elementary flexible cobordisms.

A $2 n$-dimensional Weinstein structure $(\omega, X, \phi), n \geq 2$, is called subcritical if all critical points of the function $\phi$ have index $<n$. Any subcritical Weinstein structure in dimension $2 n>4$ is by definition flexible.

Remark 5.8. The property of a Weinstein structure being subcritical is not preserved under Weinstein homotopies because one can always create a pair of critical points of index $n$ and $n-1$. It is an open problem whether or not flexibility is preserved under Weinstein homotopies.

The following results are proven in 8 using in a crucial way the theory of loose Legendrian knots.

Theorem 5.9. (1) Let $\mathfrak{W}=(V, \omega, X, \phi)$ be a flexible Weinstein manifold of dimension $2 n>4$. Then there exists a flexible Weinstein homotopy $\mathfrak{W}=$ $\left(V, \omega_{t}, X_{t}, \phi_{t}\right), t \in[0,1]$, with $\mathfrak{W}_{0}=\mathfrak{W}$, which is fixed outside a compact set and such that the Morse function $\phi_{1}$ has minimal number of critical points allowed by the Morse theory. If $\phi$ has finitely many critical points, then the homotopy can be made fixed at infinity.

(2) Let $\mathfrak{W}_{0}=\left(\omega_{0}, X_{0}, \phi_{0}\right)$ and $\mathfrak{W}_{1}=\left(\omega_{1}, X_{1}, \phi_{1}\right)$ be two flexible Weinstein structures on a manifold $V$ of dimension $2 n$. Suppose that $\omega_{0}$ and $\omega_{1}$ are homotopic as non-degenerate (not necessarily closed) 2-forms. Then $\mathfrak{W}_{0}$ and $\mathfrak{W}_{1}$ can be connected by a homotopy $\mathfrak{W}_{t}=\left(\omega_{t}, X_{t}, \phi_{t}\right), t \in[0,1]$, of flexible Weinstein structures.

An analog of Theorem 5.9 also holds for Weinstein cobordisms. Combined with Theorem [5.7, we also get an analog of Theorem [5.9 in the Stein case. We will formulate it here only for Stein cobordisms.

Theorem $5.10([8]) . \quad$ (1) Let $\mathfrak{W}=(W, J, \phi)$ be a flexible Stein cobordism of dimension $2 n>4$. Then there exists a homotopy of defining $J$-convex functions $\phi_{t}: W \rightarrow \mathbb{R}, t \in[0,1]$, with $\phi_{0}=\phi$, such that the Morse function $\phi_{1}$ has minimal number of critical points allowed by the Morse theory. 
(2) Any two flexible Stein cobordism structures $\left(W, J_{0}\right)$ and $\left(W, J_{1}\right)$ which are homotopic as almost complex structures are homotopic as flexible Stein structures.

In particular, we have the following Stein/Weinstein version of the $h$-cobordism theorem.

Corollary 5.11 (Weinstein and Stein $h$-cobordism theorem). Any flexible Weinstein structure on a product cobordism $W=Y \times[0,1]$ of dimension $2 n>4$ is homotopic to a Weinstein structure $(W, \omega, X, \phi)$, where $\phi: W \rightarrow[0,1]$ is a defining function without critical points. Similarly, any flexible Stein cobordism $(W, J)$ which is diffeomorphic to $Y \times[0,1]$ admits a defining $J$-convex function without critical points.

We note that the rigid methods allowed Seidel-Smith and McLean to show that without the flexibility assumption the above claim is wrong; see 63,83 .

5.5.3. Symplectomorphisms of flexible Weinstein manifolds. Theorem 5.9 has the following consequence for symplectomorphisms of flexible Weinstein manifolds.

Corollary 5.12. Let $\mathfrak{W}=(V, \omega, X, \phi)$ be a flexible Weinstein manifold of dimension $2 n>4$, and let $f: V \rightarrow V$ be a diffeomorphism such that $f^{*} \omega$ is homotopic to $\omega$ through non-degenerate 2 -forms. Then there exists a diffeotopy $f_{t}: V \rightarrow V$, $t \in[0,1]$, such that $f_{0}=f$, and $f_{1}$ is an exact symplectomorphism of $(V, \omega)$.

Remark 5.13. Even if $\mathfrak{W}$ is of finite type, i.e., $\phi$ has finitely many critical points, and $f=$ id outside a compact set, the diffeotopy $f_{t}$ provided by Theorem 5.12 will be in general not equal to the identity outside a compact set.

5.5.4. Equidimensional symplectic embeddings of flexible Weinstein manifolds. The following result about equidimensional symplectic embeddings of flexible Weinstein domains is proven in 32 as an application of Lagrangian caps technique.

Theorem $5.14([32])$. Let $(W, \omega, X, \phi)$ be a flexible Weinstein domain with Liouville form $\lambda$. Let $\Lambda$ be any other Liouville form on $W$ such that the symplectic forms $\omega$ and $\Omega:=d \Lambda$ are homotopic as non-degenerate (not necessarily closed) 2-forms. Then there exists an isotopy $h_{t}: W \hookrightarrow W$ such that $h_{0}=\operatorname{Id}$ and $h_{1}^{*} \Lambda=\varepsilon \lambda+d H$ for some small $\varepsilon>0$ and some smooth function $H: W \rightarrow \mathbb{R}$. In particular, $h_{1}$ defines a symplectic embedding $(W, \varepsilon \omega) \hookrightarrow(W, \Omega)$.

Corollary 5.15 ([32]). Let $(W, \omega, Z, \phi)$ be a flexible Weinstein domain, and let $(X, \Omega)$ be any symplectic manifold of the same dimension. Then any smooth embedding $f_{0}: W \hookrightarrow X$, such that the form $f_{0}^{*} \Omega$ is exact and the differential $d f$ : $T W \rightarrow T X$ is homotopic to a symplectic homomorphism, is isotopic to a symplectic embedding $f_{1}:(W, \varepsilon \omega) \hookrightarrow(X, \Omega)$ for some small $\varepsilon>0$. Moreover, if $\Omega=d \Lambda$, then the embedding $f_{1}$ can be chosen in such a way that the 1-form $f_{1}^{*} \Lambda-i_{Z} \omega$ is exact. If, moreover, the Liouville vector field dual to $\Lambda$ is complete, then the embedding $f_{1}$ exists for an arbitrarily large constant $\varepsilon$.

5.6. Topology of polynomially and rationally convex domains. We finish this section by discussing the implications of the above flexibility results for a problem of a high-dimensional complex analysis concerning the topology of polynomially and rationally convex domains. 
Polynomial, rational, and holomorphic convexity. Recall the following complex analytic notions of convexity for domains in $\mathbb{C}^{n}$. For a compact set $K \subset \mathbb{C}^{n}$, one defines its polynomial hull as

$$
\widehat{K}_{\mathcal{P}}:=\left\{z \in \mathbb{C}^{n}|| P(z)\left|\leq \max _{u \in K}\right| P(u) \mid \text { for all complex polynomials } P \text { on } \mathbb{C}^{n}\right\},
$$

and its rational hull as

$$
\widehat{K}_{\mathcal{R}}:=\left\{z \in \mathbb{C}^{n}|| R(z)\left|\leq \max _{u \in K}\right| R(u) \mid \text { for all rational functions } R=\frac{P}{Q},\left.Q\right|_{K} \neq 0\right\} .
$$

Given an open set $U \supset K$, the holomorphic hull of $K$ in $U$ is defined as

$$
\widehat{K}_{\mathcal{H}}^{U}:=\left\{z \in U|| f(z)\left|\leq \max _{u \in K}\right| f(u) \mid \text { for all holomorphic functions } f \text { on } U\right\} .
$$

A compact set $K \subset \mathbb{C}^{n}$ is called rationally (resp. polynomially) convex if $\widehat{K}_{\mathcal{R}}=$ $K$ (resp. $\left.\widehat{K}_{\mathcal{P}}=K\right)$. An open set $U \subset \mathbb{C}^{n}$ is called holomorphically convex if $\widehat{K}_{\mathcal{H}}^{U}$ is compact for all compact sets $K \subset U$. A compact set $K \subset \mathbb{C}^{n}$ is called holomorphically convex if it is the intersection of its holomorphically convex open neighborhoods. We have

polynomially convex $\Longrightarrow$ rationally convex $\Longrightarrow$ holomorphically convex.

According to a theorem of E. Levi [58, any holomorphically convex domain $W \subset \mathbb{C}^{n}$ has weakly $i$-convex (= pseudo-convex) boundary $\partial W$. The converse statement that the interior of any domain in $\mathbb{C}^{n}$ with weakly $i$-convex boundary is holomorphically convex is known as the Levi problem. It was resolved in an increasingly more general context in the series of papers begining from K. Oka's paper 73 to the paper [16 of F. Docquier and H. Grauert.

We call a domain $W \subset \mathbb{C}^{n} i$-convex if its boundary is $i$-convex. Note that any weakly $i$-convex domain in $\mathbb{C}^{n}$ can be $C^{\infty}$-approximated by a slightly smaller $i$-convex one.

Topology of polynomially and rationally convex domains. Any $i$-convex domain $W \subset \mathbb{C}^{n}$ admits a defining $i$-convex function, so in particular it admits a defining Morse function without critical points of index $>n$ (see, e.g., [8]). It follows that any holomorphically, rationally, or polynomially convex domain has the same property. We already stated above, see Theorem 4.4, that for $n \geq 3$, any domain in $\mathbb{C}^{n}$ with such a Morse function is smoothly isotopic to an $i$-convex one.

It turns out, in the spirit of Theorem 4.4 that for $n \geq 3$ there are no additional constraints on the topology of rationally convex domains.

Theorem $5.16(9])$. A compact domain $W \subset \mathbb{C}^{n}, n \geq 3$, is smoothly isotopic to a rationally convex domain if and only if it admits a defining Morse function without critical points of index $>n$.

The next result gives necessary and sufficient constraints on the topology of polynomially convex domains.

Theorem 5.17 (9]). A compact domain $W \subset \mathbb{C}^{n}, n \geq 3$, is smoothly isotopic to a polynomially convex domain if and only if it satisfies the following topological condition:

(T) $W$ admits a defining Morse function without critical points of index $>n$, and $H_{n}(W ; G)=0$ for every abelian group $G$. 
The "only if" part is well known; see 2 and also 35. Note that, in view of the universal coefficient theorem, condition $(\mathrm{T})$ is equivalent to the condition

(T') $W$ admits a defining Morse function without critical points of index $>n$, $H_{n}(W)=0$, and $H_{n-1}(W)$ has no torsion.

Further analysis of condition (T) yields

Proposition 5.18 ([9]). (a) If $W$ is simply connected, then condition ( $\mathrm{T}$ ) is equivalent to the existence of a defining Morse function without critical points of index $\geq n$.

(b) For any $n \geq 3$ there exists a (non-simply connected) domain $W$ satisfying condition $(\mathrm{T})$ with $\pi_{n}(W, \partial W) \neq 0$. In particular, $W$ does not admit a defining function without critical points of index $\geq n$.

Theorems 5.16 and 5.17 are consequences of the following more precise result for flexible Stein domains:

Theorem $5.19(9])$. Let $(W, J)$ be a flexible Stein domain of complex dimension $n \geq 3$, and let $f: W \hookrightarrow \mathbb{C}^{n}$ be a smooth embedding such that $f^{*} i$ is homotopic to $J$ through almost complex structures. Then $(W, J)$ is deformation equivalent to a rationally convex domain in $\mathbb{C}^{n}$. More precisely, $f$ is smoothly isotopic to an embedding $g: W \hookrightarrow \mathbb{C}^{n}$ such that $g(W) \subset \mathbb{C}^{n}$ is rationally convex, and $g^{*} i$ is Stein homotopic to $J$. If in addition $H_{n}(W ; G)=0$ for every abelian group $G$, then $g(W)$ can be made polynomially convex.

We note that the proofs of all the above results concerning polynomial and rational convexity are based, in addition to the theory of flexible Weinstein manifolds and analytic techniques from the book [8], on the following complex analytic characterization of the polynomial and rational convexity.

The following classical criterion for polynomial convexity goes back to K. Oka's paper [73] (see also [79, Theorem 1.3.8]).

Criterion 5.20. An i-convex domain $W \subset \mathbb{C}^{n}$ is polynomially convex if and only if there exists an exhausting $i$-convex function $\phi: \mathbb{C}^{n} \rightarrow \mathbb{R}$ such that $W=\{\phi \leq 0\}$.

To formulate a criterion for rational convexity, consider the following condition on a $J$-convex domain $W$ in a complex manifold $(X, J)$ :

(R) There exists a $J$-convex function $\phi: W \rightarrow \mathbb{R}$ such that $W=\{\phi \leq 0\}$, and the form $-d d^{\mathbb{C}} \phi$ on $W$ extends to a Kähler form $\omega$ on the whole $X$.

The following criterion for rational convexity was proved by S. Nemirovski 69. as a corollary of a result of J. Duval and N. Sibony [18, Theorem 1.1].

Criterion 5.21. An i-convex domain $W \subset \mathbb{C}^{n}$ is rationally convex if and only if it satisfies condition $(\mathrm{R})$.

The situation in complex dimension 2 is more complicated. The following theorem of S. Nemirovski and K. Siegel gives necessary and sufficient conditions for a disc bundle over a (not necessarily orientable) surface to be realizable as a rationally convex domain in $\mathbb{C}^{2}$. Recall that a 2 -disc bundle over a non-orientable surface with the orientable total space has a well-defined integer valued Euler number $e$; see [89]. 
Let $D(\chi, e)$ denote the orientable $D^{2}$-bundle over an orientable surface of Euler characteristic $\chi$, with Euler number $e$. Let $\tilde{D}(\chi, e)$ denote the non-orientable bundle over a non-orientable surface of Euler characteristic $\chi$, with Euler number $e$.

Theorem $5.22([70])$. The following manifolds can be realized as rationally convex domains in $\mathbb{C}^{2}$ :

- $D(\chi, 0)$ for $\chi \neq 2$;

- $\tilde{D}(\chi, e)$ for $(\chi, e) \neq(1,-2)$ and $e \in\{2 \chi-4,2 \chi+4, \ldots,-2 \chi-4+4[\chi / 4+1]\}$.

Moreover, these are the only possibilities.

5.7. Classification of overtwisted contact structures in all dimensions. It was recently discovered that there exists a high-dimensional analog of overtwisted contact structures (see Section 4) and that the extension $h$-principle and the corresponding classification result holds in all dimensions, not only in dimension 3.

Let us recall that a formal homotopy counterpart of a contact structure is an almost contact structure. This is a defined up to a scalar factor pair $(\lambda, \omega)$, where $\lambda$ is non-vanishing 1-form on $M$, possibly with local coefficients in a non-trivial 1bundle, and $\omega$ is a non-degenerate 2-form on the hyperplane field $\xi=\{\alpha=0\}$ with coefficients in the same local system. In the coorientable case, i.e., when $T M / \xi$ is trivial, the existence of almost contact structure is equivalent to the existence of a stable almost complex structure on $M$, i.e., a complex structure on the bundle $T M \oplus \varepsilon^{1}$, where $\varepsilon^{1}$ is the trivial line bundle over $M$.

We already mentioned above that in 1969 M. Gromov [45] proved a parametric $h$-principle for contact structures on an open manifold $M$ : any almost contact structure is homotopic to a genuine one, and two contact structures are homotopic if they are homotopic as almost contact structures.

For 3-dimensional closed manifolds J. Martinet 61] and R. Lutz 60] proved the non-parametric existence $h$-principle for 3 -manifolds. Then D. Bennequin [4] discovered a first contact rigidity result: the 1-parametric $h$-principle fails for contact structures on $S^{3}$. As was already mentioned in Section 4. building on Bennequin's work, in 23 the author introduced a dichotomy of 3-dimensional contact manifolds into tight and overtwisted and established a parametric $h$-principle for overtwisted contact structures.

A lot of progress was achieved in the last three decades in the direction of contact rigidity. Tight contact structures were also classified on several classes of $3-$ manifolds; see e.g. 26, 42,53,54. V. Colin, E. Giroux, and K. Honda in [11 proved that any atoroidal contact 3-manifold admits at most finitely many non-isotopic tight contact structures. Symplectic Field Theory provided powerful invariants for distinguishing contact structures on manifolds of all dimensions; see [29, 85], 63], et al.

The flexible side also had a number of success stories. In particular, the existence $h$-principle for a closed 5-manifold was gradually established, beginning from the work of H. Geiges [36, 37] and H. Geiges and C.B. Thomas, 39, 40] and followed by the work of R. Casals, D. M. Pancholi, and F. Presas [10] and J. Etnyre 33. For manifolds of dimension $>5$ the results were more scarce. The work [24] implied existence of contact structures on all closed $(2 n+1)$-dimensional, $n>1$, manifolds which bound manifolds of homotopy type of $(n+1)$-dimensional cell complexes. F. Bourgeois [7] proved, using work of E. Giroux [41, that for any closed contact 


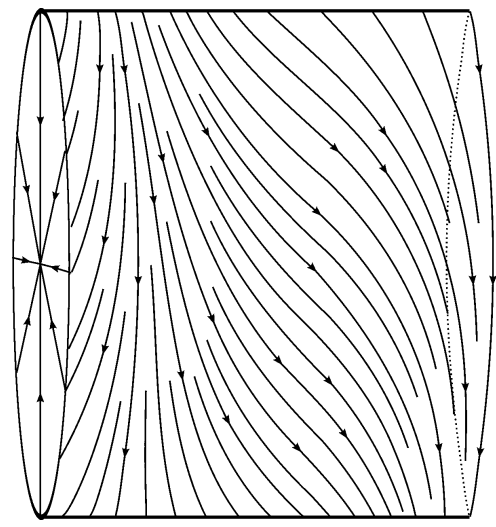

FIGURE 5.2. An overtwisted disk with its characteristic foliation

manifold $M$ and any surface $\Sigma$ with genus at least one, the product $M \times \Sigma$ admits a contact structure. This positively answered a long-standing problem about existence of contact structures on tori of dimension $2 n+1>5$.

The following recently proved results significantly advanced the flexible side of the contact story in higher dimensions.

Theorem $5.23([\underline{6})$. Let $M$ be a $(2 n+1)$-manifold, and let $A \subset M$ be a closed set. Suppose that $M$ and $M \backslash A$ are connected. Let $\xi$ be an almost contact structure on $M$ which is genuine on $\mathcal{O} p A \subset M$. Then $\xi$ is homotopic relative to $A$ to a genuine contact structure. In particular, any almost contact structure on a closed manifold is homotopic to a genuine contact structure.

It turned out that the overtwisting phenomenon can be generalized to higher dimensions as well. Without giving a precise definition, we will just say here that a contact manifold $(M, \xi)$ is called overtwisted if it admits a contact embedding of a piecewise smooth $2 n$-disc $D_{\text {ot }}$ with a certain model germ $\zeta_{\text {ot }}$ of a contact structure; see Figure 5.2 .

Theorem 5.24 ([6]). On any closed manifold $M$ any almost contact structure is homotopic to an overtwisted contact structure which is unique up to isotopy.

To formulate a more precise result consider a $(2 n+1)$-dimensional manifold $M$, a closed subset $A$, and an almost contact structure $\xi_{0}$ on $M$ that is a genuine contact structure on $A$, and define $\operatorname{Cont}_{\mathrm{ot}}\left(M ; A, \xi_{0}\right)$ to be the space of contact structures on $M$ that are overtwisted on $M \backslash A$ and that coincide with $\xi_{0}$ on $\mathcal{O} p A$. The notation $\operatorname{cont}\left(M ; A, \xi_{0}\right)$ stands for the corresponding formal object, i.e., the space of almost contact structures that agree with $\xi_{0}$ on $\mathcal{O} p A$. We denote by $j$ the inclusion map $j: \operatorname{Cont}_{\mathrm{ot}}\left(M ; A, \xi_{0}\right) \rightarrow \operatorname{cont}\left(M ; A, \xi_{0}\right)$. We denote by $\operatorname{Cont}_{\mathrm{ot}}\left(M ; A, \xi_{0}, \phi\right)$ and $\operatorname{cont}_{\mathrm{ot}}\left(M ; A, \xi_{0}, \phi\right)$ the subspaces of $\operatorname{Cont}_{\mathrm{ot}}\left(M ; A, \xi_{0}\right)$ and $\operatorname{cont}\left(M ; A, \xi_{0}\right)$ of contact and almost contact structures for which a fixed embedding $\phi: D_{\text {ot }} \rightarrow M \backslash A$ is a contact embedding $\left(D_{\mathrm{ot}}, \zeta_{\mathrm{ot}}\right) \rightarrow(M, \xi)$.

Theorem 5.25 ([6]). If $M \backslash A$ is connected, then the inclusion map induces an isomorphism

$$
j_{*}: \pi_{0}\left(\operatorname{Cont}_{\mathrm{ot}}\left(M ; A, \xi_{0}\right)\right) \rightarrow \pi_{0}\left(\operatorname{cont}\left(M ; A, \xi_{0}\right)\right),
$$


and moreover the map

$$
j: \operatorname{Cont}_{\mathrm{ot}}\left(M ; A, \xi_{0}, \phi\right) \rightarrow \operatorname{cont}_{\mathrm{ot}}\left(M ; A, \xi_{0}, \phi\right)
$$

is a (weak) homotopy equivalence.

As an application of Theorem 5.24 we provide an explicit classification of overtwisted contact structures on spheres $S^{2 n+1}$. Note that almost contact structures on the sphere $S^{2 n+1}$ are classified by the homotopy group $\pi_{2 n+1}(S O(2 n+2) / U(n+1))$. Hence, Theorem 5.24 implies that the elements of this group also enumerate isotopy classes of overtwisted contact structures on $S^{2 n+1}$.

The following lemma computes this group.

Lemma 5.26 (Bruno Harris, [52]).

$$
\pi_{2 n+1}(S O(2 n+2) / U(n+1))= \begin{cases}\mathbb{Z} / n ! \mathbb{Z}, & n=4 k ; \\ \mathbb{Z}, & n=4 k+1 ; \\ \mathbb{Z} / \frac{n !}{2} \mathbb{Z}, & n=4 k+2 ; \\ \mathbb{Z} \oplus \mathbb{Z} / 2 \mathbb{Z}, & n=4 k+3 .\end{cases}
$$

In particular, on spheres $S^{8 k+1}, k>0$, there are exactly $(4 k)$ ! different overtwisted contact structures, on spheres $S^{8 k+5}, k \geq 0$, there are $\frac{(4 k+2) !}{2}$ different overtwisted contact structures, while on all other spheres there are infinitely many. For instance, there is a unique overtwisted contact structure on $S^{5}$.

It is interesting to note that $S^{5}$ has infinitely many tight, i.e., non-overtwisted contact structures. Besides the standard contact structure, these are examples given by Brieskorn spheres (see [85). The full classification of tight contact structures on any manifold of dimension $>3$ is an open problem.

We note that there were many proposals for defining the overtwisting phenomenon in dimension greater than three. The overtwistedness in the above sense is stronger than any other possible notions. Namely, any exotic phenomenon, e.g., a plastikstufe [71, can be found in any overtwisted contact manifold. Indeed, suppose we are given some exotic model $(A, \zeta)$ which is compact contact manifold with boundary which formally embeds into $\left(M, \xi_{\text {ot }}\right)$. Given any smooth embedding $A \hookrightarrow M$, there is an almost contact structure on $M$ which is equal to $\zeta$ on $A$ and homotopic to $\xi_{\text {ot }}$ through almost contact structures. Theorem 5.23 implies this almost contact structure can be homotoped relative to $A$ to a contact structure $\xi$ on $M$. Theorem 5.25 and Gray's theorem [44 implies that $\zeta$ is isotopic to $\xi_{\text {ot }}$.

In particular, the known results about contact manifolds with a plastikstufe apply to overtwisted manifolds as well:

- overtwisted contact manifolds are not symplectically fillable [71;

- the Weinstein conjecture holds for any contact form defining an overtwisted contact structure on a closed manifold [1];

- any Legendrian submanifold whose complement is overtwisted is loose. Conversely, any loose Legendrian in an overtwisted ambient manifold has an overtwisted complement [66].

We also point out the following corollary concerning iso-contact embeddings into an overtwisited contact manifold

- Let $(N, \zeta)$ and $(M, \xi)$ be two contact manifolds, so that $(M, \xi)$ is overtwisted and either $N$ is open or $\operatorname{dim} N<\operatorname{dim} M$. Let $\Phi: T N \rightarrow T M$ be an isocontact bundle homomorphism (i.e., $\Phi_{*} \zeta \subset \xi$ respects the linear conformal 
symplectic structure) covering an embedding $f: N \rightarrow M$ such that $d f, \Phi$ : $T N \rightarrow T M$ are homotopic as injective bundle homomorphisms. Then $f$ is isotopic to an iso-contact embedding $\widetilde{f}:(N, \zeta) \rightarrow(M, \xi)$.

\section{NeXt StePs OF SyMPLECTIC FleXibiLity}

6.1. Holomorphic curves or nothing dichotomy. Since Gromov's seminal paper [46] holomorphic curve technique, sometimes applied in a very subtle and unexpected way, brought tremendous progress on the side of symplectic rigidity. In fact, all other methods and techniques, such as generating functions, Floer homological techniques etc., are different variations on the theme of holomorphic curves. The multiple attempts to introduce genuinely different methods so far did not bring to life anything promising.

Hence, I propose the "holomorphic curves or nothing" principle: If one cannot disprove a flexible h-principle type conjecture using holomorphic curve techniques and its derivatives, then it should be true. In fact, this principle guided the authors in the work [6] because there was no feasible way to use holomorphic curves in overtwisted contact manifods to establish any rigidity results.

Here are a few very optimistic conjectures implied by the above principle.

6.2. Symplectic structures on closed manifolds. Let us recall Problem 3.1(1): When does a germ of a symplectic or contact structure along the boundary of an $n$-ball $B$ extend to $B$ ? In the contact case Theorem 5.25 provides a flexible answer to this question.

In the symplectic case there is, first of all, an additional volume obstruction. Namely, given a germ $\omega$ of a symplectic structure along a boundary $\partial D^{2 n}$ of a ball $D^{2 n}, n>1$, we observe that the form $\omega$ is exact, $\omega=d \eta$, and if $\omega$ extends as a symplectic form to $D^{2 n}$, then by Stokes' theorem

$$
\int_{\partial D^{2 n}} \eta \wedge \omega^{n-1}=\int_{D^{2 n}} \omega^{n}>0,
$$

where the left-hand side is independent of the choice of a primitive $\eta$. But even with this modification, an extension $h$-principle for symplectic structures fails in all dimensions $>1$, as a holomorphic curve argument, similar to Gromov's proof of his famous non-squeezing theorem, [46, shows. It seems that this argument never appeared in print, so we sketch it here. In the standard symplectic space

$$
\left(\mathbb{R}^{2 n}, \omega_{\text {st }}=d p_{1} \wedge d q_{1}+\cdots+d p_{n} \wedge d q_{n}\right),
$$

consider three parallelepipeds

$$
\begin{aligned}
& A:=\left\{\left|p_{i}\right|,\left|q_{i}\right| \leq 1, i=1, \ldots, n-1,\left|q_{n}\right| \leq 1,0 \leq p_{n} \leq 1\right\}, \\
& B:=\left\{\left|p_{i}\right|,\left|q_{i}\right| \leq 2, i=1, \ldots, n-1,\left|q_{n}\right| \leq 2,0 \leq p_{n} \leq \frac{1}{4}\right\},
\end{aligned}
$$

and

$$
C:=\left\{\left|p_{i}\right|,\left|q_{i}\right| \leq 1, i=1, \ldots, n-1,\left|q_{n}\right| \leq 1,0 \leq p_{n} \leq \frac{1}{8}\right\} .
$$

Note that Volume $A=2^{2 n-1}<$ Volume $B=4^{2 n-2}$, while $A$ has a larger Gromov's width: $w(A)=2>w(B)=1$. Hence, Gromov's non-squeezing theorem implies that there is no symplectic embedding $A \rightarrow B$. The parallelepipeds $A, B$, and $C$ share a common boundary piece $E:=\left\{p_{n}=0,\left|p_{i}\right|,\left|q_{i}\right| \leq 1, i=1, \ldots, n-1,, 0 \leq\right.$ $\left.p_{n} \leq 1\right\}$, so that the remaining parts of the boundary of $A$ and $B$ form an immersed 
piecewise smooth $(2 n-1)$-sphere $\widetilde{\Sigma}:=(\partial A \backslash \operatorname{Int} E) \cup(\partial B \backslash \operatorname{Int} E)$. Similarly, the remaining parts of the boundary of $C$ and $B$ form an embedded piecewise smooth $(2 n-1)$-sphere $\Sigma:=(\partial C \backslash \operatorname{Int} E) \cup(\partial B \backslash \operatorname{Int} E)$ which bounds a ball $G$. There exists a smooth immersion $h: \mathcal{O} p \Sigma \rightarrow \mathcal{O} p \widetilde{\Sigma}$ such that $h(\Sigma)=\widetilde{\Sigma}$. We claim that the symplectic structure $h^{*} \omega$ on $\mathcal{O} p \Sigma$ does not extend as a symplectic structure to $G$ while neither homotopic nor volume obstructions exist for such an extension. Indeed, suppose that such an extension $\widetilde{\omega}$ does exist. Then one can glue back $(A, \omega)$ to $(G, \widetilde{\omega})$ using a symplectomorphism $h$ on $\mathcal{O} p(\partial A \backslash E)$. The resulting symplectic manifold

$$
(H, \widehat{\omega}):=(G, \widetilde{\omega}) \underset{h}{\cup}(A, \omega)
$$

contains the parallelepiped $(A, \omega)$, while near its boundary $\partial H$ it is symplectomorphic to $(B, \omega)$. It remains to notice that the proof of Gromov's non-squeezing theorem works without any changes for symplectic embeddings into any aspherical symplectic manifold which is symplectomorphic to a polydisc near its boundary. Hence, the symplectic structure $h^{*} \omega$ on $\mathcal{O} p \Sigma$ is not extendable to $G$.

The existence $h$-principle for symplectic structures on closed manifolds is also known to be wrong in dimension 4; see 84. However, in higher dimensions there are no known obstructions, and even no feasible approaches how such obstruction could be constructed. Could it be then that this problem in dim $>4$ belongs to the flexible world? In other words,

Conjecture 6.1. On any manifold $M$ of dimension $n=2 k>4$ with a cohomology class $\eta \in H^{2}(M)$ with $\eta^{k} \neq 0$ and a non-degenerate 2 -form $\omega_{0}$, there exists a symplectic form $\omega$ homotopic to $\omega_{0}$ through non-degenerate forms, whose cohomology class $[\omega]$ can be deformed to $\eta$ keeping its $k$-th power non-vanishing.

As we already pointed out above, the analogous statement is wrong in dimension 4. However, could it be that it is still virtually true?

Conjecture 6.2. Given any 4-manifold $M$ with a cohomology class $\eta \in H^{2}(M ; \mathbb{Z})$ with $\eta^{2} \neq 0$ and a non-degenerate 2 -form $\omega_{0}$, one can find an orientable surface $F \subset M$ which realizes a homology class dual to $N \eta$ for a sufficiently large $N$, and such that a certain branch cover of $M$ along $F$ admits a symplectic structure for which the branching locus is symplectic, whose cohomology class is the pullback of $\eta$ and which is homotopic to the pullback of the 2-form $\omega_{0}$ through non-degenerate forms.

6.3. Symplectic caps. Holomorphic curve methods also seem to be helpless for symplectic manifolds with an overtwisted concave contact boundary. We say that a compact symplectic manifold $(X, \omega)$ has a concave contact boundary $(Y, \xi)$ if near $Y$ the form $\omega$ is exact: $\omega=d \lambda$, the contact structure $\xi$ is defined by the form $\left.\lambda\right|_{Y}$, and the Liouville vector field $Z$ which is $\omega$-dual to $\lambda, \iota(Z) \omega=\lambda$, is inward transverse to $Y=\partial X$.

Conjecture 6.3. Symplectic manifolds with a concave overtwisted contact boundary satisfy an h-principle. In particular, suppose we are given

- a compact $2 n$-dimensional manifold $X$ with boundary $Y$;

- a non-degenerate (not necessarily closed) 2-form $\omega$ which is exact near $Y$;

- an inward transverse to $Y$ Liouville vector field $Z$ for $\left.\omega\right|_{\mathcal{O}_{p} Y}$, i.e., $\omega=$ $d(\lambda:=\iota(Z) \omega)$ on $\mathcal{O} p Y$, such that the contact structure $\xi=\left\{\left.\lambda\right|_{Y}=0\right\}$ is overtwisted; 
- a closed 2-form $\eta$ on $X$ such that $\left.\eta\right|_{O p Y}=\left.\omega\right|_{\mathcal{O p}_{Y}}$ and

$$
\int_{X} \eta^{n}>\int_{Y} \lambda \wedge(d \lambda)^{n-1} .
$$

Then there exists a homotopy $\omega_{t}, t \in[0,1]$, connecting $\omega_{0}=\omega$ and a symplectic form $\omega_{1}$, such that $\omega_{t}$ is non-degenerate on $X$, and $\omega_{t}=\omega$ on $\mathcal{O} p Y$ for all $t \in[0,1]$.

Conjecture 6.3 would imply the following weaker version of Conjecture 6.1 which potentially could hold also in dimension 4 .

Conjecture 6.4. Under assumptions of Conjecture 6.1, the required symplectic form $\omega$ on $X$ could be constructed with just one singular point, so that near this point $\omega$ is symplectomorphic to the negative part of the symplectization of an overtwisted contact structure on $S^{2 n-1}$ from the standard almost contact homotopy class.

We note that according to Theorem 5.24 such a contact structure is unique. A similar $h$-principle may even hold for symplectic cobordisms with an overtwisted concave boundary (or even more optimistically, for the symplectic extension problem). In particular, it seems plausible that there exists a symplectic concordance, i.e., a symplectic structure on $S^{2 n-1} \times[0,1], n>1$, between the overtwisted contact structure on the concave side $S^{2 n-1} \times 0$ and the standard one on the convex one $S^{2 n-1} \times 1$.

Note that for $n=2$, a symplectic cobordism (rather that a concordance) does exist between an overtwisted contact sphere on the concave side and the standard contact sphere on the convex one; see, e.g., 34.

6.4. How far could Arnold conjectures go? The most optimistic form of the Arnold conjecture concerning the lower bound for the number of intersection points of an exact Lagrangian submanifold $L \subset T^{*} M$ of a closed manifold $M$ with its 0 section asserts that the number of intersection points, assuming the transversality of the intersection, is bounded below by the minimal number $\operatorname{Morse}(M)$ of critical points of a Morse function on $M$. However, the best lower bound that is known in the case when $L$ is Lagrangian isotopic to the 0 -section is in terms of the stable Morse number Morse $_{\mathrm{st}}(M)$, i.e., the minimal number of critical points of a function on $M \times \mathbb{R}^{q}$ which, outside a compact set, coincides with the pullback of a nondegenerate quadratic form on $\mathbb{R}^{q}$. The numbers $\operatorname{Morse}(M)$ and $\operatorname{Morse}_{\text {st }}(M)$ are known to be different (see, e.g., [14). It does not seem feasible to the author that holomorphic curve methods could be used to prove an estimate in terms of Morse $(M)$. Hence, the "holomorphic curves or nothing" dichotomy suggests that if $\operatorname{Morse}(M)>\operatorname{Morse}_{\text {st }}(M)$, then there should exist an exact Lagrangian submanifold $L \subset T^{*}(M)$, possibly even Hamiltonian isotopic to the 0 -section which intersects $M$ transversely in less than $\operatorname{Morse}(M)$ points.

\section{ABOUt THE AUThOR}

Yakov Eliashberg is the Herald L. and Caroline L. Ritch Professor of Mathematics at Stanford University. His work belongs to symplectic and contact geometry and topology and their interaction with complex analysis. 


\section{REFERENCES}

[1] Peter Albers and Helmut Hofer, On the Weinstein conjecture in higher dimensions, Comment. Math. Helv. 84 (2009), no. 2, 429-436, DOI 10.4171/CMH/167. MR2495800 (2010b:53152)

[2] Aldo Andreotti and Raghavan Narasimhan, A topological property of Runge pairs, Ann. of Math. (2) 76 (1962), 499-509. MR0140714 (25 \#4128)

[3] Vladimir Arnol'd, Sur une propriété topologique des applications globalement canoniques de la mécanique classique (French), C. R. Acad. Sci. Paris 261 (1965), 3719-3722. MR.0193645 (33 \#1861)

[4] Daniel Bennequin, Entrelacements et équations de Pfaff (French), Third Schnepfenried geometry conference, Vol. 1 (Schnepfenried, 1982), Astérisque, vol. 107, Soc. Math. France, Paris, 1983, pp. 87-161. MR753131 (86e:58070)

[5] G.D. Birkhoff, Proof of Poincaré's geometric theorem, Trans. Amer. Math. Soc., 14(1913), $14-22$

[6] S. Borman, Y. Eliashberg and E. Murphy, Existence and classification of overtwisted contact structures in all dimensions, arXiv:1404.6157.

[7] Frédéric Bourgeois, Odd dimensional tori are contact manifolds, Int. Math. Res. Not. 30 (2002), 1571-1574, DOI 10.1155/S1073792802205048. MR 1912277 (2003f:53157)

[8] Kai Cieliebak and Yakov Eliashberg, From Stein to Weinstein and back, American Mathematical Society Colloquium Publications, vol. 59, American Mathematical Society, Providence, RI, 2012. Symplectic geometry of affine complex manifolds. MR 3012475

[9] K. Cieliebak and Y. Eliashberg, The topology of rationally and polynomially convex domains, Invent. Math., 2014, DOI 10.1007/s00222-014-0511-6.

[10] R. Casals, D. Pancholi and F. Presas, Almost contact 5-folds are contact, 2012, arXiv:1203.2166

[11] Vincent Colin, Emmanuel Giroux, and Ko Honda, On the coarse classification of tight contact structures, Topology and geometry of manifolds (Athens, GA, 2001), Proc. Sympos. Pure Math., vol. 71, Amer. Math. Soc., Providence, RI, 2003, pp. 109-120, DOI 10.1090/pspum/071/2024632. MR2024632 (2005a:53146)

[12] C. C. Conley and E. Zehnder, The Birkhoff-Lewis fixed point theorem and a conjecture of V. I. Arnol'd, Invent. Math. 73 (1983), no. 1, 33-49, DOI 10.1007/BF01393824. MR707347 (85e:58044)

[13] S. K. Donaldson, Symplectic submanifolds and almost-complex geometry, J. Differential Geom. 44 (1996), no. 4, 666-705. MR1438190 (98h:53045)

[14] Mihai Damian, On the stable Morse number of a closed manifold, Bull. London Math. Soc. 34 (2002), no. 4, 420-430, DOI 10.1112/S0024609301008955. MR:1897421 (2003b:57050)

[15] S. K. Donaldson, Lefschetz pencils on symplectic manifolds, J. Differential Geom. 53 (1999), no. 2, 205-236. MR1802722 (2002g:53154)

[16] Ferdinand Docquier and Hans Grauert, Levisches Problem und Rungescher Satz für Teilgebiete Steinscher Mannigfaltigkeiten (German), Math. Ann. 140 (1960), 94-123. MR0148939 (26 \#6435)

[17] T. Ekholm and I. Smith, Exact Lagrangian immersions with a single double point, preprint, arXiv:1111.5932.

[18] Julien Duval and Nessim Sibony, Polynomial convexity, rational convexity, and currents, Duke Math. J. 79 (1995), no. 2, 487-513, DOI 10.1215/S0012-7094-95-07912-5. MR.1344768 (96f:32016)

[19] Tobias Ekholm and Ivan Smith, Exact Lagrangian immersions with one double point revisited, Math. Ann. 358 (2014), no. 1-2, 195-240, DOI 10.1007/s00208-013-0958-6. MR.3157996

[20] Tobias Ekholm, Yakov Eliashberg, Emmy Murphy, and Ivan Smith, Constructing exact Lagrangian immersions with few double points, Geom. Funct. Anal. 23 (2013), no. 6, 1772-1803, DOI 10.1007/s00039-013-0243-6. MR.3132903

[21] Y. Eliashberg, Rigidity of symplectic structures, preprint 1981.

[22] Ya. M. Eliashberg, A theorem on the structure of wave fronts and its application in symplectic topology (Russian), Funktsional. Anal. i Prilozhen. 21 (1987), no. 3, 65-72, 96. MR911776 (88j:58033)

[23] Y. Eliashberg, Classification of overtwisted contact structures on 3-manifolds, Invent. Math. 98 (1989), no. 3, 623-637, DOI 10.1007/BF01393840. MR.1022310 (90k:53064) 
[24] Yakov Eliashberg, Topological characterization of Stein manifolds of dimension > 2, Internat. J. Math. 1 (1990), no. 1, 29-46, DOI 10.1142/S0129167X90000034. MR.1044658 (91k:32012)

[25] Yakov Eliashberg, Filling by holomorphic discs and its applications, Geometry of lowdimensional manifolds, 2 (Durham, 1989), London Math. Soc. Lecture Note Ser., vol. 151, Cambridge Univ. Press, Cambridge, 1990, pp. 45-67. MR.1171908 (93g:53060)

[26] Yakov Eliashberg, Contact 3-manifolds twenty years since J. Martinet's work (English, with French summary), Ann. Inst. Fourier (Grenoble) 42 (1992), no. 1-2, 165-192. MR1162559 (93k:57029)

[27] Y. Eliashberg, Recent progress in symplectic flexibility, The Influence of Solomon Lefschetz in Geometry and Topology: 50 years of Mathematics in CINVESTAV, (L. Katzarkov, E. Lupercio, and F. Turrubiates, eds.), vol. 621, Contemp. Math., American Mathematical Society, Providence, RI, 2014.

[28] Yakov Eliashberg and Maia Fraser, Topologically trivial Legendrian knots, J. Symplectic Geom. 7 (2009), no. 2, 77-127. MR2496415(2010d:57024)

[29] Y. Eliashberg, A. Givental, and H. Hofer, Introduction to symplectic field theory, Geom. Funct. Anal. Special Volume (2000), 560-673, DOI 10.1007/978-3-0346-0425-3_4. GAFA 2000 (Tel Aviv, 1999). MR.1826267 (2002e:53136)

[30] Yakov Eliashberg and Mikhael Gromov, Convex symplectic manifolds, Several complex variables and complex geometry, Part 2 (Santa Cruz, CA, 1989), Proc. Sympos. Pure Math., vol. 52, Amer. Math. Soc., Providence, RI, 1991, pp. 135-162. MR1128541 (93f:58073)

[31] Y. Eliashberg and N. Mishachev, Introduction to the h-principle, Graduate Studies in Mathematics, vol. 48, American Mathematical Society, Providence, RI, 2002. MR.1909245 (2003g:53164)

[32] Yakov Eliashberg and Emmy Murphy, Lagrangian caps, Geom. Funct. Anal. 23 (2013), no. 5, 1483-1514, DOI 10.1007/s00039-013-0239-2. MR3102911

[33] J. Etnyre, Contact structures on 5-manifolds, 2012, arXiv:1210.5208.

[34] John B. Etnyre and Ko Honda, On connected sums and Legendrian knots, Adv. Math. 179 (2003), no. 1, 59-74, DOI 10.1016/S0001-8708(02)00027-0. MR2004728(2004h:57005)

[35] Franc Forstnerič, Complements of Runge domains and holomorphic hulls, Michigan Math. J. 41 (1994), no. 2, 297-308, DOI 10.1307/mmj/1029004997. MR.1278436 (95k:32014)

[36] Hansjörg Geiges, Contact structures on 1-connected 5-manifolds, Mathematika 38 (1991), no. 2, 303-311 (1992), DOI 10.1112/S002557930000663X. MR1147828 (93e:57042)

[37] Hansjörg Geiges, Applications of contact surgery, Topology 36 (1997), no. 6, 1193-1220, DOI 10.1016/S0040-9383(97)00004-9. MR1452848 (98d:57044)

[38] Hansjörg Geiges, An introduction to contact topology, Cambridge Studies in Advanced Mathematics, vol. 109, Cambridge University Press, Cambridge, 2008. MR2397738(2008m:57064)

[39] H. Geiges and C. B. Thomas, Contact topology and the structure of 5-manifolds with $\pi_{1}=Z_{2}$ (English, with English and French summaries), Ann. Inst. Fourier (Grenoble) 48 (1998), no. 4, 1167-1188. MR.1656012 (2000a:57069)

[40] Hansjörg Geiges and Charles B. Thomas, Contact structures, equivariant spin bordism, and periodic fundamental groups, Math. Ann. 320 (2001), no. 4, 685-708, DOI 10.1007/PL00004491. MR:1857135(2002k:57061)

[41] Emmanuel Giroux, Géométrie de contact: de la dimension trois vers les dimensions supérieures (French, with French summary), Proceedings of the International Congress of Mathematicians, Vol. II (Beijing, 2002), Higher Ed. Press, Beijing, 2002, pp. 405-414. MR.1957051 (2004c:53144)

[42] Emmanuel Giroux, Structures de contact en dimension trois et bifurcations des feuilletages de surfaces (French), Invent. Math. 141 (2000), no. 3, 615-689, DOI 10.1007/s002220000082. MR,1779622(2001i:53147)

[43] A. B. Givental', Lagrangian imbeddings of surfaces and the open Whitney umbrella (Russian), Funktsional. Anal. i Prilozhen. 20 (1986), no. 3, 35-41, 96. MR868559 (88g:58018)

[44] John W. Gray, Some global properties of contact structures, Ann. of Math. (2) 69 (1959), 421-450. MR0112161 (22 \#3016)

[45] M. L. Gromov, Stable mappings of foliations into manifolds (Russian), Izv. Akad. Nauk SSSR Ser. Mat. 33 (1969), 707-734. MR0263103 (41 \#7708)

[46] M. Gromov, Pseudoholomorphic curves in symplectic manifolds, Invent. Math. 82 (1985), no. 2, 307-347, DOI 10.1007/BF01388806. MR809718 (87j:53053) 
[47] M. L. Gromov, Stable mappings of foliations into manifolds (Russian), Izv. Akad. Nauk SSSR Ser. Mat. 33 (1969), 707-734. MR0263103 (41 \#7708)

[48] Mikhael Gromov, Partial differential relations, Ergebnisse der Mathematik und ihrer Grenzgebiete (3) [Results in Mathematics and Related Areas (3)], vol. 9, Springer-Verlag, Berlin, 1986. MR864505 (90a:58201)

[49] Larry Guth, Symplectic embeddings of polydisks, Invent. Math. 172 (2008), no. 3, 477-489, DOI 10.1007/s00222-007-0103-9. MR2393077 (2008k:53195)

[50] Dmitry Fuchs and Serge Tabachnikov, Invariants of Legendrian and transverse knots in the standard contact space, Topology 36 (1997), no. 5, 1025-1053, DOI 10.1016/S00409383(96)00035-3. MR1445553 (99a:57006)

[51] Kenji Fukaya, Yong-Geun Oh, Hiroshi Ohta, and Kaoru Ono, Lagrangian intersection Floer theory: anomaly and obstruction. Part I, AMS/IP Studies in Advanced Mathematics, vol. 46, American Mathematical Society, Providence, RI; International Press, Somerville, MA, 2009. MR2553465(2011c:53217)

[52] Bruno Harris, Some calculations of homotopy groups of symmetric spaces, Trans. Amer. Math. Soc. 106 (1963), 174-184. MR0143216 (26 \#776)

[53] Ko Honda, On the classification of tight contact structures. I, Geom. Topol. 4 (2000), 309368, DOI 10.2140/gt.2000.4.309. MR1786111 (2001i:53148)

[54] Ko Honda, On the classification of tight contact structures. II, J. Differential Geom. 55 (2000), no. 1, 83-143. MR1849027 (2002g:53155)

[55] Morris W. Hirsch, Immersions of manifolds, Trans. Amer. Math. Soc. 93 (1959), 242-276. MR0119214(22 \#9980)

[56] H. Hofer, Pseudoholomorphic curves in symplectizations with applications to the Weinstein conjecture in dimension three, Invent. Math. 114 (1993), no. 3, 515-563, DOI 10.1007/BF01232679. MR.1244912 (94j:58064)

[57] Nicolaas H. Kuiper, On $C^{1}$-isometric imbeddings. I, II, Nederl. Akad. Wetensch. Proc. Ser. A. $\mathbf{5 8}$ = Indag. Math. 17 (1955), 545-556, 683-689. MR0075640 (17,782c)

[58] E. Levi, Studii sui punti singolari essenziali delle funzioni analitiche di due o più variabili complesse, Annali di Mat oura ed appl. 17, 61-87 (1910).

[59] P. Lisca and G. Matić, Tight contact structures and Seiberg-Witten invariants, Invent. Math. 129 (1997), no. 3, 509-525, DOI 10.1007/s002220050171. MR1465333 (98f:57055)

[60] Robert Lutz, Structures de contact sur les fibrés principaux en cercles de dimension trois (French, with English summary), Ann. Inst. Fourier (Grenoble) 27 (1977), no. 3, ix, 1-15. MR0478180 (57 \#17668)

[61] J. Martinet, Formes de contact sur les variétés de dimension 3 (French), Proceedings of Liverpool Singularities Symposium, II (1969/1970), Springer, Berlin, 1971, pp. 142-163. Lecture Notes in Math., Vol. 209. MR0350771 (50 \#3263)

[62] W. S. Massey, Proof of a conjecture of Whitney, Pacific J. Math. 31 (1969), 143-156. MR.0250331(40 \#3570)

[63] Mark McLean, Lefschetz fibrations and symplectic homology, Geom. Topol. 13 (2009), no. 4, 1877-1944, DOI 10.2140/gt.2009.13.1877. MR2497314(2011d:53224)

[64] J. Milnor, Whitehead torsion, Bull. Amer. Math. Soc. 72 (1966), 358-426. MR0196736 (33 \#4922)

[65] Jürgen Moser, On the volume elements on a manifold, Trans. Amer. Math. Soc. 120 (1965), 286-294. MR0182927 (32 \#409)

[66] Emmy Murphy, Klaus Niederkrüger, Olga Plamenevskaya, and András I. Stipsicz, Loose Legendrians and the plastikstufe, Geom. Topol. 17 (2013), no. 3, 1791-1814, DOI 10.2140/gt.2013.17.1791. MR3073936

[67] E. Murphy, Loose Legendrian embeddings in high dimensional contact manifolds, arXiv:1201.2245.

[68] John Nash, $C^{1}$ isometric imbeddings, Ann. of Math. (2) 60 (1954), 383-396. MR0065993 $(16,515 \mathrm{e})$

[69] S. Yu. Nemirovski1, Finite unions of balls in $\mathbb{C}^{n}$ are rationally convex (Russian), Uspekhi Mat. Nauk 63 (2008), no. 2(380), 157-158, DOI 10.1070/RM2008v063n02ABEH004518; English transl., Russian Math. Surveys 63 (2008), no. 2, 381-382. MR2640558(2011f:32021)

[70] S. Nemirovski and K. Siegel, Rationally convex domains and singular Lagrangian surfaces, in preparation. 
[71] Klaus Niederkrüger, The plastikstufe - a generalization of the overtwisted disk to higher dimensions, Algebr. Geom. Topol. 6 (2006), 2473-2508, DOI 10.2140/agt.2006.6.2473. MR2286033 (2007k:57053)

[72] K. Niederkruger and O. van Koert, Every Contact Manifolds can be given a Nonfillable Contact Structure, Int. Math. Res. Notices, 2009, 4463-4479.

[73] Kiyoshi Oka, Sur les fonctions analytiques de plusieurs variables. IX. Domaines finis sans point critique intérieur (French), Jap. J. Math. 23 (1953), 97-155 (1954). MR0071089 $(17,82 \mathrm{~b})$

[74] H. Poincaré, Sur une théorème de géométrie, Rend. Circ. Mat. Palermo 33 (1912), 375-507.

[75] Lee Rudolph, An obstruction to sliceness via contact geometry and "classical" gauge theory, Invent. Math. 119 (1995), no. 1, 155-163, DOI 10.1007/BF01245177. MR.1309974 (95k:57013)

[76] S. Smale, On the structure of manifolds, Amer. J. Math. 84 (1962), 387-399. MR0153022 (27 \#2991)

[77] Stephen Smale, The classification of immersions of spheres in Euclidean spaces, Ann. of Math. (2) 69 (1959), 327-344. MR0105117 (21 \#3862)

[78] Denis Sauvaget, Curiosités lagrangiennes en dimension 4 (French, with English and French summaries), Ann. Inst. Fourier (Grenoble) 54 (2004), no. 6, 1997-2020 (2005). MR2134231 (2006c:53087)

[79] Edgar Lee Stout, Polynomial convexity, Progress in Mathematics, vol. 261, Birkhäuser Boston, Inc., Boston, MA, 2007. MR2305474(2008d:32012)

[80] Clifford Henry Taubes, The Seiberg-Witten equations and the Weinstein conjecture, Geom. Topol. 11 (2007), 2117-2202, DOI 10.2140/gt.2007.11.2117. MR2350473(2009b:57055)

[81] Leonid Polterovich, Monotone Lagrange submanifolds of linear spaces and the Maslov class in cotangent bundles, Math. Z. 207 (1991), no. 2, 217-222, DOI 10.1007/BF02571385. MR.1109663 (93c:58075)

[82] L. Polterovich, The surgery of Lagrange submanifolds, Geom. Funct. Anal. 1 (1991), no. 2, 198-210, DOI 10.1007/BF01896378. MR.1097259 (93d:57062)

[83] Paul Seidel and Ivan Smith, The symplectic topology of Ramanujam's surface, Comment. Math. Helv. 80 (2005), no. 4, 859-881, DOI 10.4171/CMH/37. MR2182703 (2006j:53127)

[84] Clifford Henry Taubes, The Seiberg-Witten invariants and symplectic forms, Math. Res. Lett. 1 (1994), no. 6, 809-822, DOI 10.4310/MRL.1994.v1.n6.a15. MR1306023 (95j:57039)

[85] Ilya Ustilovsky, Infinitely many contact structures on $S^{4 m+1}$, Internat. Math. Res. Notices 14 (1999), 781-791, DOI 10.1155/S1073792899000392. MR1704176 (2000f:57028)

[86] Claude Viterbo, A proof of Weinstein's conjecture in $\mathbf{R}^{2 n}$ (English, with French summary), Ann. Inst. H. Poincaré Anal. Non Linéaire 4 (1987), no. 4, 337-356. MR917741 (89d:58048)

[87] C. T. C. Wall, Geometrical connectivity. I, J. London Math. Soc. (2) 3 (1971), 597-604. MR0290387 (44 \#7569a)

[88] Alan Weinstein, Contact surgery and symplectic handlebodies, Hokkaido Math. J. 20 (1991), no. 2, 241-251, DOI 10.14492/hokmj/1381413841. MR.1114405 (92g:53028)

[89] Hassler Whitney, On the topology of differentiable manifolds, Lectures in Topology, University of Michigan Press, Ann Arbor, Mich., 1941, pp. 101-141. MR0005300(3,133a)

Department of Mathematics, Stanford University, Stanford, California 94305 